\title{
HARDY-LITTLEWOOD TYPE INEQUALITIES FOR LAGUERRE SERIES
}

\section{CHIN-CHENG LIN and SHU-HUEY LIN}

Received 30 August 2001

Let $\left\{c_{j}\right\}$ be a null sequence of bounded variation. We give appreciate smoothness and growth conditions on $\left\{c_{j}\right\}$ to obtain the pointwise convergence as well as $L^{r}$-convergence of Laguerre series $\sum c_{j} \mathscr{L}_{j}^{a}$. Then, we prove a Hardy-Littlewood type inequality $\int_{0}^{\infty}|f(t)|^{r} d t \leq$ $C \sum_{j=0}^{\infty}\left|c_{j}\right|^{r} \bar{j}^{1-r / 2}$ for certain $r \leq 1$, where $f$ is the limit function of $\sum c_{j} \mathscr{L}_{j}^{a}$. Moreover, we show that if $f(x) \sim \sum c_{j} \mathscr{L}_{j}^{a}$ is in $L^{r}, r \geq 1$, we have the converse Hardy-Littlewood type inequality $\sum_{j=0}^{\infty}\left|c_{j}\right|^{r} \bar{j}^{\beta} \leq C \int_{0}^{\infty}|f(t)|^{r} d t$ for $r \geq 1$ and $\beta<-r / 2$.

2000 Mathematics Subject Classification: 42C10, 42C15.

1. Introduction. Given complex numbers $\left\{c_{j}\right\}_{j \in \mathbb{Z}}$ satisfying $\sum\left|c_{j}\right|^{r}(|j|+1)^{r-2}<\infty$ for some $r \geq 2$, Hardy and Littlewood [4] (see also [14, Theorem 3.19, page 109]) proved in 1926 that $c_{j}$ 's are the Fourier coefficients of an $f$ in $L^{r}$, and

$$
\int_{0}^{2 \pi}|f(t)|^{r} d t \leq A_{r} \sum_{j=-\infty}^{\infty}\left|c_{j}\right|^{r}(|j|+1)^{r-2} .
$$

Also, they proved that if $f(x) \sim \sum c_{j} e^{i j x}$ is in $L^{r}, 1<r \leq 2$, then

$$
\sum_{j=-\infty}^{\infty}\left|c_{j}\right|^{r}(|j|+1)^{r-2} \leq A_{r} \int_{0}^{2 \pi}|f(t)|^{r} d t .
$$

Later on, Paley [10] (see also [14, Theorem 5.1, page 121]) extended Hardy and Littlewood's results to general systems of orthonormal and uniformly bounded functions over an interval. In this paper, we concentrate on the Laguerre system, and prove the similar inequalities

$$
\begin{gathered}
\int_{0}^{\infty}|f(t)|^{r} d t \leq C \sum_{j=0}^{\infty}\left|c_{j}\right|^{r} \bar{j}^{1-r / 2} \quad \text { for certain } r \leq 1, \\
\sum_{j=0}^{\infty}\left|c_{j}\right|^{r} \bar{j}^{\beta} \leq C \int_{0}^{\infty}|f(t)|^{r} d t \quad \text { for } r \geq 1, \beta<-\frac{r}{2},
\end{gathered}
$$

where $\bar{\xi}$ means $\max \{\xi, 1\}$.

For $a>-1$, the Laguerre polynomials of type $a$ are defined by the formula

$$
L_{n}^{a}(t)=\frac{1}{n !} t^{-a} e^{t} \frac{d^{n}}{d t^{n}}\left(t^{n+a} e^{-t}\right), \quad n=0,1,2, \ldots
$$


Each $L_{n}^{a}$ is a polynomial of degree $n$, whose explicit expression is

$$
L_{n}^{a}(t)=\sum_{k=0}^{n} \frac{(-1)^{k}}{k !}\left(\begin{array}{l}
n+a \\
n-k
\end{array}\right) t^{k} .
$$

The Laguerre polynomials form a complete orthogonal system in $L^{2}\left(\mathbb{R}^{+}, t^{a} e^{-t} d t\right)$. Hence, if we define $\mathscr{L}_{n}^{a}(t)$ by

$$
\mathscr{L}_{n}^{a}(t)=\sqrt{\frac{n !}{\Gamma(n+a+1)}} e^{-t / 2} t^{a / 2} L_{n}^{a}(t),
$$

then they form an orthonormal basis in $L^{2}\left(\mathbb{R}^{+}, d t\right)$ with the inner product $\langle f, g\rangle=$ $\int_{0}^{\infty} f(t) g(t) d t$.

A number of authors have studied the problems of pointwise convergence and mean convergence for different types of Laguerre series. Of particular interest are the results of Askey and Wainger [1], Chen and Lin [2], Długosz [3], Muckenhoupt $[7,8,9]$, and Stempak $[11,12,13]$. However, all of them started at a given function $f$ to get the Laguerre coefficients $\left\{c_{j}\right\}$, and proved the pointwise convergence or mean convergence of the series $\sum c_{j} \mathscr{L}_{j}^{a}$. In this paper, we start from $\left\{c_{j}\right\}$ satisfying

$$
\begin{gathered}
\sum_{j=0}^{\infty}\left|\Delta^{p} c_{j}\right| \bar{j}^{p / 2-1 / 4}<\infty, \\
\left|c_{j}\right| \bar{j}^{p / 2-1 / 4}(\overline{\log j})^{1+\epsilon}=O(1) \quad \text { as } j \rightarrow \infty,
\end{gathered}
$$

for some $p \in \mathbb{N}$ and $\epsilon>0$, and prove the pointwise convergence of Laguerre series $\sum c_{j} \mathscr{L}_{j}^{a}$. Here, $\Delta^{p} c_{j}$ denotes the finite-order difference

$$
\Delta^{0} c_{j}=c_{j}, \quad \Delta^{p} c_{j}=\Delta^{p-1} c_{j}-\Delta^{p-1} c_{j+1} \quad \text { for } p \in \mathbb{N} .
$$

Then, we strengthen the assumptions on $\left\{c_{j}\right\}$ such that the Laguerre series $\sum c_{j} \mathscr{L}_{j}^{a}$ converges not only pointwise but also in $L^{r}$-metric. In addition, we obtain the HardyLittlewood type inequalities.

THEOREM 1.1. Let $a \geq 0$. Assume that $\left\{c_{j}: j \geq 0\right\}$ satisfies

$$
\begin{gathered}
\sum_{j=0}^{\infty}\left|\Delta^{p} c_{j}\right|^{r} j^{1-r / 2}<\infty, \\
\left|c_{j}\right| \bar{j}^{2 / r-3 / 2+\epsilon}=O(1) \quad \text { as } j \rightarrow \infty,
\end{gathered}
$$

for some $p \in \mathbb{N}, \epsilon>0$, and $r \leq \min \{1,4 /(1+2 p)\}$. Then, the Laguerre series $\sum c_{j} \mathscr{L}_{j}^{a}(t)$ converges to $f \in L^{r}\left(\mathbb{R}^{+}\right)$pointwise and in $L^{r}$-metric, where

$$
f(t)=e^{-t / 2} t^{a / 2} \sum_{j=0}^{\infty}\left(\Delta^{p} b_{j}\right) L_{j}^{a+p}(t)
$$

and $b_{j}=c_{j} \sqrt{j ! / \Gamma(j+a+1)}$. 
COROLlaRY 1.2. Under the same assumptions of Theorem 1.1, there is a constant $C$ independent of $f$ such that

$$
\int_{0}^{\infty}|f(t)|^{r} d t \leq C \sum_{j=0}^{\infty}\left|c_{j}\right|^{r} \bar{j}^{1-r / 2} .
$$

We also prove the above converse inequality in the following theorem.

THEOREM 1.3. Let $a \geq 0$. If $f \in L^{r}\left(\mathbb{R}^{+}\right), r \geq 1$, then there is a constant $C$ independent of $f$, such that

$$
\sum_{j=0}^{\infty}\left|c_{j}\right|^{r} \bar{j}^{\beta} \leq C \int_{0}^{\infty}|f(t)|^{r} d t \quad \forall \beta<-\frac{r}{2},
$$

where $c_{j} \equiv \int_{0}^{\infty} f(t) \mathscr{L}_{j}^{a}(t) d t$.

REMARK 1.4. For $1 \leq r<4 / 3$, we can find a $\beta<-r / 2$ such that $\beta>r-2$. Thus, Theorem 1.3 improves Paley's result for Laguerre system $\left\{\mathscr{L}_{j}^{a}\right\}$. Moreover, Kanjin [5] showed that, for $f(t) \sim \sum_{j=0}^{\infty} c_{j} \mathscr{L}_{j}^{a}(t)$ in $H^{1}\left(\mathbb{R}^{+}\right), \sum_{j=0}^{\infty} c_{j}(j+1)^{-1} \leq C\|f\|_{H^{1}\left(\mathbb{R}^{+}\right)}$, which is the special case of Theorem 1.3 for $r=1$ and $\beta=-1$.

In the next section, we first give some estimates of Laguerre functions and talk about the pointwise convergence and $L^{r}$-convergence of Laguerre series. Then we prove Corollary 1.2 and Theorem 1.3 in Section 3. Finally, we mention that $C$, possibly with subscripts, denotes a constant which may stand for a different number from one appearance to another.

2. Pointwise convergence and mean convergence. It is known that the Laguerre functions satisfy the estimates

$$
\left|\mathscr{L}_{j}^{a}(t)\right| \leq \begin{cases}C t^{a / 2} v^{a / 2}, & \text { if } 0 \leq t \leq \frac{1}{v} \\ C t^{-1 / 4} v^{-1 / 4}, & \text { if } \frac{1}{v}<t \leq \frac{v}{2} \\ C v^{-1 / 4}\left(v^{1 / 3}+|t-v|\right)^{-1 / 4}, & \text { if } \frac{v}{2}<t \leq \frac{3 v}{2} \\ C e^{-\gamma t}, & \text { if } \frac{3 v}{2}<t<\infty\end{cases}
$$

where $v=4 j+2 a+2$, and both $C$ and $\gamma$ are positive constants independent of $j$ and $t$ (cf. $[1,9])$. Hence, by a straightforward calculation, we have

$$
\left|L_{j}^{\alpha}(t)\right| \leq C e^{t / 2} t^{-\alpha / 2-1 / 4} \bar{j}^{\alpha / 2-1 / 4}(1+t)^{1 / 6}
$$

for all $j \geq 0$, all $t \geq 0$, and $\alpha=a, a+1, \ldots, a+p$. Also,

$$
\int_{0}^{\infty}\left|t^{-p / 2} \mathscr{L}_{j}^{a+p}(t)\right|^{r} d t \leq C \bar{j}^{1-r / 2-p r / 2}
$$


for $r / 2+p r \leq 2, r \neq 4, a>-2 / r$, and all $j \geq 0$. In particular, $\left\|\mathscr{L}_{j}^{a}\right\|_{L^{r}\left(\mathbb{R}^{+}\right)}^{r} \leq C \bar{j}^{1-r / 2}$ for $0<r<4, a>-2 / r$, and all $j \geq 0$. As to $r \geq 4$, it follows from [6, Lemma 1] that, for $a \geq 0$,

$$
\left\|\mathscr{L}_{j}^{a}\right\|_{L^{r}\left(\mathbb{R}^{+}\right)}= \begin{cases}O\left(j^{1 / r-1 / 2}\right), & \text { for } 1 \leq r<4 \\ O\left(j^{1 / r-1 / 2}(\log j)^{1 / r}\right), & \text { for } r=4 ; \\ O\left(j^{-1 / r}\right), & \text { for } r>4 .\end{cases}
$$

Let $s_{n}(t)$ denote the partial sums of Laguerre series defined by

$$
s_{n}(t)=\sum_{j=0}^{n} c_{j} \mathscr{L}_{j}^{a}(t)
$$

Set $b_{j}=c_{j} \sqrt{j ! / \Gamma(j+a+1)}$. Then

$$
s_{n}(t)=e^{-t / 2} t^{a / 2} \sum_{j=0}^{n} b_{j} L_{j}^{a}(t) .
$$

For $t>0$ and $n \in \mathbb{N}$, the well-known equation

$$
L_{j}^{a+1}(t)-L_{j+1}^{a+1}(t)=-L_{j+1}^{a}(t)
$$

and the summation by parts yield

$$
s_{n}(t)=e^{-t / 2} t^{a / 2}\left(\sum_{j=0}^{n}\left(\Delta b_{j}\right) L_{j}^{a+1}(t)+b_{n+1} L_{n}^{a+1}(t)\right) .
$$

Repeating the same process, we get

$$
\begin{aligned}
s_{n}(t) & =e^{-t / 2} t^{a / 2} \sum_{j=0}^{n}\left(\Delta^{p} b_{j}\right) L_{j}^{a+p}(t)+e^{-t / 2} t^{a / 2} \sum_{j=0}^{p-1}\left(\Delta^{j} b_{n+1}\right) L_{n}^{a+j+1}(t) \\
& \equiv I_{1}(t)+I_{2}(t) .
\end{aligned}
$$

Using the inequality $1-\sqrt{1-y} \leq y$ for $y \in[0,1]$, we have

$$
\left|\Delta^{k}\left(\sqrt{\frac{j !}{\Gamma(j+a+1)}}\right)\right| \leq C_{p} \sqrt{\frac{j !}{\Gamma(j+a+1)}} \frac{a}{j+a+1} \leq a C_{p} \bar{j}^{-a / 2-1} \quad \text { for } 1 \leq k \leq p,
$$

which with Leibniz's rule implies

$$
\begin{aligned}
\left|\Delta^{p} b_{j}\right| & =\left|\Delta^{p}\left(c_{j} \sqrt{\frac{j !}{\Gamma(j+a+1)}}\right)\right| \\
& \leq \sqrt{\frac{(j+p) !}{\Gamma(j+p+a+1)}}\left|\Delta^{p} c_{j}\right|+C_{a p} \bar{j}^{-a / 2-1} \sum_{i=0}^{p-1}\left(\begin{array}{c}
p \\
i
\end{array}\right)\left|\Delta^{i} c_{j}\right| \\
& \leq C_{a p}\left\{\bar{j}^{-a / 2}\left|\Delta^{p} c_{j}\right|+\bar{j}^{-a / 2-1}\left(\max _{j \leq k \leq j+p-1}\left|c_{k}\right|\right)\right\} .
\end{aligned}
$$


Thus,

$$
\left|I_{1}(t)\right| \leq C_{a p} e^{-t / 2} t^{a / 2} \sum_{j=0}^{n}\left\{\bar{j}^{-a / 2}\left|\Delta^{p} c_{j}\right|+\bar{j}^{-a / 2-1}\left(\max _{j \leq k \leq j+p-1}\left|c_{k}\right|\right)\right\}\left|L_{j}^{a+p}(t)\right| .
$$

Condition (1.8) says that the inequality $\left|c_{j}\right| \bar{j}^{p / 2-1 / 4} \leq C(\overline{\log j})^{-1-\epsilon}$ holds for all $j$ 's with sufficiently large $C$. Hence, conditions (1.7), (1.8), and estimate (2.2) yield

$$
\begin{aligned}
\left|I_{1}(t)\right| & \leq C_{t} \sum_{j=0}^{n}\left\{\bar{j}^{-a / 2}\left|\Delta^{p} c_{j}\right|+\bar{j}^{-a / 2-1}\left(\max _{j \leq k \leq j+p-1}\left|c_{k}\right|\right)\right\} \bar{j}^{(a+p) / 2-1 / 4} \\
& \leq C_{t} \sum_{j=0}^{n} \bar{j}^{p / 2-1 / 4}\left|\Delta^{p} c_{j}\right|+\sum_{j=0}^{\infty} \bar{j}^{-1} \max _{j \leq k \leq j+p-1} \bar{k}^{p / 2-1 / 4}\left|c_{k}\right| \\
& \leq C_{t}\left(\sum_{j=0}^{n} \bar{j}^{p / 2-1 / 4}\left|\Delta^{p} c_{j}\right|+\sum_{j=0}^{\infty} \frac{1}{\bar{j}(\overline{\log j})^{1+\epsilon}}\right) \\
& <\infty \quad \forall n \in \mathbb{N} .
\end{aligned}
$$

On the other hand, (1.8), (2.2), and the equality

$$
\Delta^{j} b_{n}=\sum_{i=0}^{j}\left(\begin{array}{l}
j \\
i
\end{array}\right)(-1)^{i} b_{n+i}
$$

imply

$$
\begin{aligned}
\left|I_{2}(t)\right| & \leq e^{-t / 2} t^{a / 2} \sum_{j=0}^{p-1}\left\{\sum_{i=0}^{j}\left(\begin{array}{l}
j \\
i
\end{array}\right)(n+1+i)^{-a / 2}\left|c_{n+1+i}\right|\right\}\left|L_{n}^{a+j+1}(t)\right| \\
& \leq C_{a p} \sum_{j=0}^{p-1}\left\{\sum_{i=0}^{j}\left(\begin{array}{l}
j \\
i
\end{array}\right)(n+1+i)^{(j+1) / 2-1 / 4}\left|c_{n+1+i}\right| t^{-(j+1) / 2-1 / 4}(1+t)^{1 / 6}\right\} \\
& \leq C_{a p} \sup _{k>n}\left\{k^{p / 2-1 / 4}\left|c_{k}\right|\right\} \sum_{j=0}^{p-1} t^{-(j+1) / 2-1 / 4}(1+t)^{1 / 6} \\
& \longrightarrow 0 \text { as } n \rightarrow \infty .
\end{aligned}
$$

Hence, $s_{n}(t)$ converges pointwise to

$$
f(t) \equiv e^{-t / 2} t^{a / 2} \sum_{j=0}^{\infty}\left(\Delta^{p} b_{j}\right) L_{j}^{a+p}(t)
$$

provided (1.7) and (1.8) hold. Hence, we have the following lemma.

LEMma 2.1. Let $a \geq 0$. Assume that $\left\{c_{j}: j \geq 0\right\}$ satisfies conditions (1.7) and (1.8). Then, the Laguerre series $\sum c_{j} \mathscr{L}_{j}^{a}(t)$ converges pointwise to the function $f(t)$ in (2.16), $t \in \mathbb{R}^{+}$. 
Now we are ready to prove Theorem 1.1 . Since $r \leq 1$ and $r / 2+p r \leq 2, p / 2-1 / 4 \leq$ $2 / r-3 / 2$ which says that (1.11) is stronger than (1.8). Also, we have

$$
\sum_{j=0}^{\infty}\left(\left|\Delta^{p} c_{j}\right| j^{p / 2-1 / 4}\right)^{r} \leq \sum_{j=0}^{\infty}\left|\Delta^{p} c_{j}\right|^{r} j^{1-r / 2} .
$$

Thus, condition (1.10) yields

$$
\sum_{j=0}^{\infty}\left(\left|\Delta^{p} c_{j}\right| j^{p / 2-1 / 4}\right)^{r}<\infty,
$$

which implies the validity of (1.7) since $\ell^{r} \subseteq \ell^{1}$. By Lemma 2.1, we get the pointwise convergence.

To finish the proof of Theorem 1.1, we still need to check its $L^{r}$-convergence. From (2.9) and inequality

$$
\|g+h\|_{r}^{r} \leq\|g\|_{r}^{r}+\|h\|_{r}^{r} \quad \text { for } 0<r \leq 1
$$

we have

$$
\begin{aligned}
\int_{0}^{\infty}\left|s_{n}(t)-f(t)\right|^{r} d t \leq & \sum_{j=n+1}^{\infty} \int_{0}^{\infty}\left\{e^{-t / 2} t^{a / 2}\left|\left(\Delta^{p} b_{j}\right) L_{j}^{a+p}(t)\right|\right\}^{r} d t \\
& +\sum_{j=0}^{p-1} \int_{0}^{\infty}\left\{e^{-t / 2} t^{a / 2}\left|\left(\Delta^{j} b_{n+1}\right) L_{n}^{a+j+1}(t)\right|\right\}^{r} d t \\
& \equiv I_{3}+I_{4} .
\end{aligned}
$$

The definition of $\mathscr{L}_{j}^{a}$, (1.10), (1.11), (2.3), and (2.11) give us

$$
\begin{aligned}
I_{3} & \leq C_{a p} \sum_{j=n+1}^{\infty}\left|j^{a / 2} \Delta^{p} b_{j}\right|^{r} j^{p r / 2} \int_{0}^{\infty}\left|t^{-p / 2} \mathscr{L}_{j}^{a+p}(t)\right|^{r} d t \\
& \leq C_{a p} \sum_{j=n+1}^{\infty}\left(\left|\Delta^{p} c_{j}\right|+j^{-1} \max _{j \leq k \leq j+p-1}\left|c_{k}\right|\right)^{r} j^{1-r / 2} \\
& \longrightarrow 0 \text { as } n \longrightarrow \infty, \\
I_{4} & \leq C_{a p} \sum_{j=0}^{p-1}\left|n^{a / 2} \Delta^{j} b_{n+1}\right|^{r} n^{(j+1) r / 2} \int_{0}^{\infty}\left|t^{-(j+1) / 2} \mathscr{L}_{n}^{a+j+1}(t)\right|^{r} d t \\
& \leq C_{a p} \max _{n \leq k \leq n+p}\left|c_{k}\right|^{r} n^{1-r / 2} \\
& \longrightarrow 0 \quad \text { as } n \rightarrow \infty .
\end{aligned}
$$

Hence, Theorem 1.1 follows immediately.

3. Proofs of Hardy-Littlewood type inequalities. From the previous arguments, conditions (1.10) and (1.11) imply that the series $\sum c_{j} \mathscr{L}_{j}^{a}(t)$ converges pointwise and in $L^{r}$-metric to $f(t)$. We show the Hardy-Littlewood type inequalities as follows. 
Proof OF COROLlary 1.2. The hypotheses of Corollary 1.2, the monotone convergence theorem, (2.4), and (2.19) can be used to show that

$$
\begin{aligned}
\int_{0}^{\infty}|f(t)|^{r} d t & =\int_{0}^{\infty}\left|\sum_{j=0}^{\infty} c_{j} \mathscr{L}_{j}^{a}(t)\right|^{r} d t \\
& \leq \sum_{j=0}^{\infty}\left|c_{j}\right|^{r} \int_{0}^{\infty}\left|\mathscr{L}_{j}^{a}(t)\right|^{r} d t \\
& \leq C \sum_{j=0}^{\infty}\left|c_{j}\right|^{r} \bar{j}^{1-r / 2} .
\end{aligned}
$$

Proof OF TheOREM 1.3. Let $f \in L^{r}\left(\mathbb{R}^{+}\right), r \geq 1$, and $c_{j}=\int_{0}^{\infty} f(t) \mathscr{L}_{j}^{a}(t) d t$. Hölder's inequality and (2.4) yield

$$
\begin{array}{rlr}
\left|c_{j}\right|^{r} \bar{j}^{\beta} & =\left|\int_{0}^{\infty} f(t) \mathscr{L}_{j}^{a}(t) d t\right|^{r} \bar{j}^{\beta} & \\
& \leq\|f\|_{r}^{r}\left(\int_{0}^{\infty}\left|\mathscr{L}_{j}^{a}(t)\right|^{r^{\prime}} d t\right)^{r / r^{\prime}} \bar{j}^{\beta} & \\
& \leq \begin{cases}C\|f\|_{L^{r\left(\mathbb{R}^{+}\right)}}^{r} \bar{j}^{r / r^{\prime}-r / 2+\beta}, & \text { for } r>\frac{4}{3}, \\
C\|f\|_{L^{r\left(\mathbb{R}^{+}\right)}}^{r} \bar{j}^{-1 / 3+\beta}(\log \bar{j})^{1 / 3}, & \text { for } r=\frac{4}{3}, \\
C\|f\|_{L^{r\left(\mathbb{R}^{+}\right)}}^{r} \bar{j}^{-r / r^{\prime}+\beta}, & \text { for } r<\frac{4}{3},\end{cases}
\end{array}
$$

where $1 / r+1 / r^{\prime}=1$. Since $\beta<-r / 2$ implies $r / r^{\prime}-r / 2+\beta<-1$,

$$
\sum_{j=0}^{\infty}\left|c_{j}\right|^{r} \bar{j}^{\beta} \leq C\|f\|_{r}^{r} .
$$

\section{REFERENCES}

[1] R. Askey and S. Wainger, Mean convergence of expansions in Laguerre and Hermite series, Amer. J. Math. 87 (1965), 695-708.

[2] C.-P. Chen and C.-C. Lin, Almost everywhere convergence of Laguerre series, Studia Math. 109 (1994), no. 3, 291-301.

[3] J. Długosz, Almost everywhere convergence of some summability methods for Laguerre series, Studia Math. 82 (1985), no. 3, 199-209.

[4] G. H. Hardy and J. E. Littlewood, Some new properties of Fourier constants, Math. Ann. 97 (1926), 159-209.

[5] Y. Kanjin, Hardy's inequalities for Hermite and Laguerre expansions, Bull. London Math. Soc. 29 (1997), no. 3, 331-337.

[6] C. Markett, Mean Cesàro summability of Laguerre expansions and norm estimates with shifted parameter, Anal. Math. 8 (1982), no. 1, 19-37.

[7] B. Muckenhoupt, Poisson integrals for Hermite and Laguerre expansions, Trans. Amer. Math. Soc. 139 (1969), 231-242.

[8]__ Mean convergence of Hermite and Laguerre series. I, Trans. Amer. Math. Soc. 147 (1970), 419-431. 
[9]___ Mean convergence of Hermite and Laguerre series. II, Trans. Amer. Math. Soc. 147 (1970), 433-460.

[10] R. E. A. C. Paley, Some theorems on orthogonal functions, Studia Math. 3 (1931), 226-238.

[11] K. Stempak, Mean summability methods for Laguerre series, Trans. Amer. Math. Soc. 322 (1990), no. 2, 671-690.

[12] _ Almost everywhere summability of Laguerre series, Studia Math. 100 (1991), no. 2, 129-147.

[13] _ Almost everywhere summability of Laguerre series. II, Studia Math. 103 (1992), no. 3, 317-327.

[14] A. Zygmund, Trigonometric Series: Vols. I, II, 2nd ed., Cambridge University Press, London, 1968.

Chin-Cheng Lin and Shu-Huey Lin: Department of Mathematics, National Central University, ChUNG-LI, TAIWAN 320, RePUblic OF CHINA 


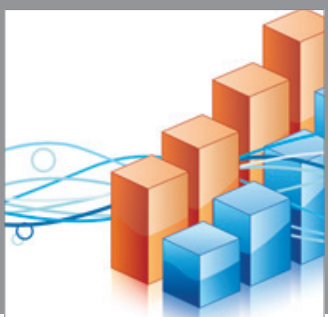

Advances in

Operations Research

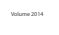

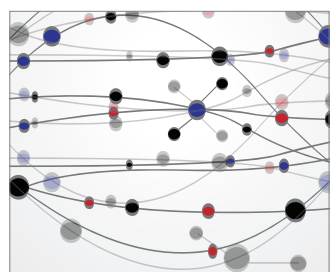

\section{The Scientific} World Journal
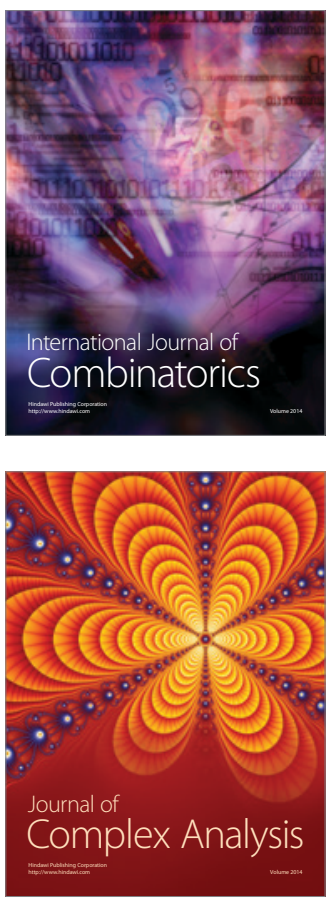

International Journal of

Mathematics and

Mathematical

Sciences
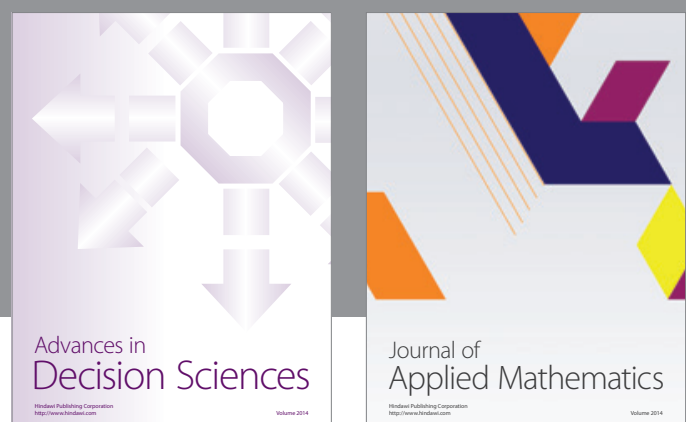

Journal of

Applied Mathematics
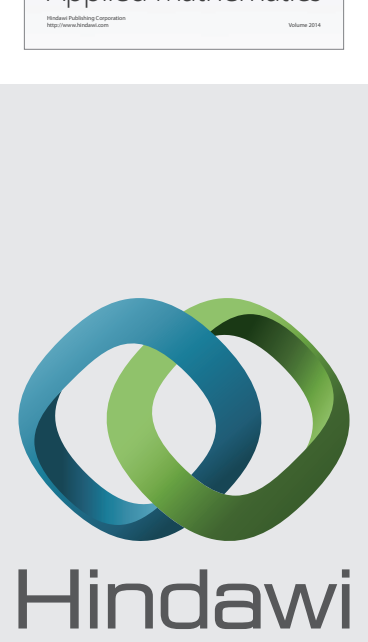

Submit your manuscripts at http://www.hindawi.com
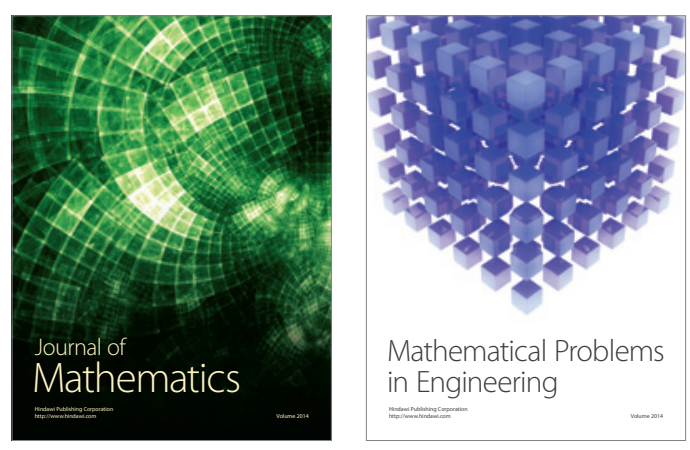

Mathematical Problems in Engineering
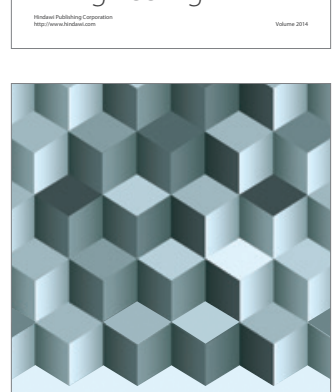

Journal of

Function Spaces
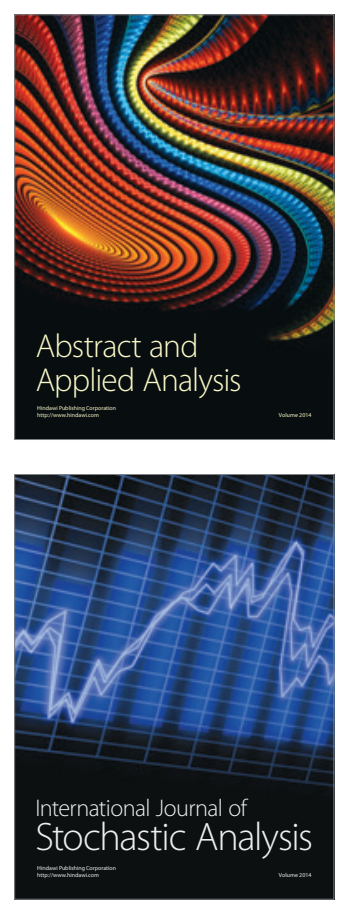

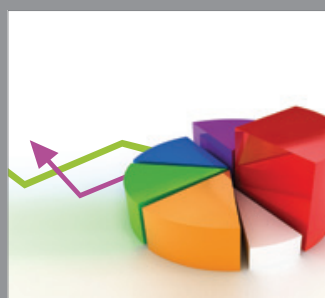

ournal of

Probability and Statistics

Promensencen
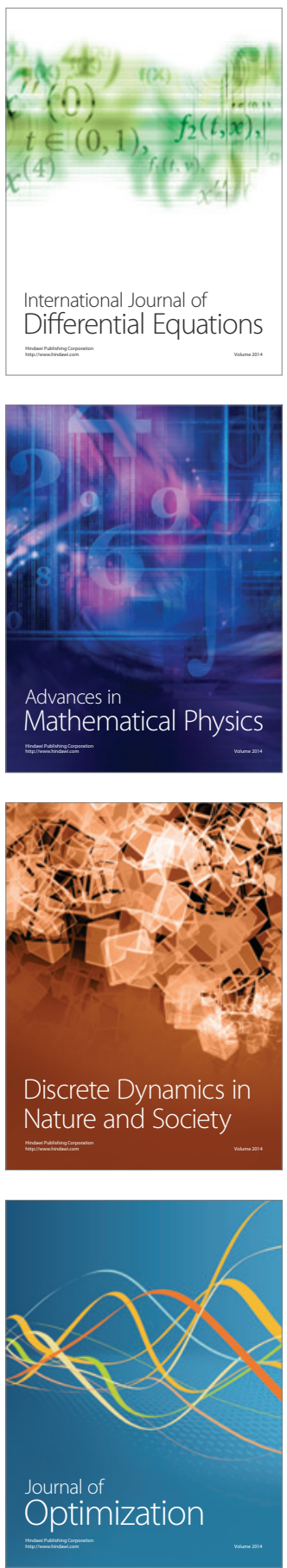